\title{
Liberación mínimamente invasiva del túnel del carpo sin instrumentación especial. Técnica quirúrgica y serie de casos
}

\author{
Minimally invasive carpal tunnel release without special \\ instrumentation. Surgical technique and case series
}

\author{
Efraín Farías Cisneros, ${ }^{*}$ Ranulfo Romo Rodríguez, ${ }^{*}$ Marisol Limón Muñoz, ${ }^{\ddagger}$ Tuna Özyurekoglu§
}

\section{RESUMEN}

Introducción: El síndrome del túnel del carpo es la neuropatía compresiva más frecuente, cuyas manifestaciones clínicas principales incluyen parestesias y disestesias en el territorio inervado por el nervio mediano en la mano. Existen varias técnicas quirúrgicas para tratar este problema con alta prevalencia a nivel mundial, en las que el ligamento transverso del carpo se incide en su totalidad. Las técnicas mínimamente invasivas han demostrado diversas ventajas sobre la técnica convencional, entre las que se incluyen una cicatriz más pequeña, ausencia de cuerda del arco palmar de los tendones flexores y evitar el contacto directo del nervio mediano sobre la cicatriz cutánea. Objetivo: Presentar una técnica de mínima invasión que no requiere instrumentación especial, así como revisar los resultados clínicos de pacientes tratados con dicha técnica. Material y métodos: Se trata de un estudio de serie de casos, retrospectivo. Ocho casos, tratados con la técnica de liberación del túnel del carpo sin instrumentación especial. Con un seguimiento de ocho semanas. Se realizaron evaluaciones de Weber y Quick-DASH, pre- y postoperatorias. Resultados: Fueron analizados con estadísticas descriptivas y t de Student, según fue necesario. En todos los casos hubo mejoría clínica. Dos de ellos

\section{ABSTRACT}

Introduction: The carpal tunnel syndrome is the most common compressive neuropathy. Its clinical presentation includes paresthesias and dysesthesias in the territory supplied by the median nerve in the hand. There are several techniques available to treat this world-wide high-prevalence syndrome, in all of them the carpal transverse ligament is completely sectioned. The minimally invasive techniques, have shown several advantages over the conventional technique, including a smaller scar, absence of palmar bowstringing of the flexor tendons, and avoidance of a direct contact of the median nerve with the skin's scar. Objective: The aim of the study was to present a minimally invasive technique for carpal tunnel release that does not require special instrumentation. We also aimed to review the clinical outcomes of patients treated with this technique. Material and methods: The study is a retrospective case series study. Eight cases treated with a carpal tunnel release technique without special instrumentation. With an eight-week follow-up. Preoperative and postoperative Weber test and Quick-DASH questionnaire were assessed. The results were analyzed with descriptive statistics and $t$-test, as needed. Results: In all cases there was clinical improvement. Two cases
* Cirujano de Mano y Ortopedista, Centro Médico ABC.

‡ Anestesióloga Regional, Instituto Nacional de Rehabilitación «Luis Guillermo Ibarra".

$\S$ Cirujano de Mano y Ortopedista, Presidente del Christine M. Kleinert Institute for the Hand and Microsurgery.

\section{Recibido para publicación: 29/01/2020. Aceptado: 03/03/2020.}

Correspondencia: Dr. Efraín Farías Cisneros

Av. Carlos Graef Fernández Núm. 154,

Centro Médico ABC, Campus Santa Fe, Torre Central,

Consultorio 104-105, Cuajimalpa, 05300, CDMX.

Tels: 5596 6155; 55966178 www.manointegral.com

E-mail: drefrain@drefrainfarias.com

Abreviaturas:

AINEs = Antiinflamatorios no esteroideos

$\mathrm{APL}=$ Abductor pollicis longus .

$\mathrm{DE}=$ Desviación estándar.

$\mathrm{EPB}=$ Extensor pollicis brevis.

$\mathrm{FCU}=$ Flexor carpi ulnaris

$I C=$ Intervalo de confianza.

LTC = Ligamento transverso del carpo.

$\mathrm{PL}=$ Palmaris longus.

STC $=$ Síndrome del túnel del carpo. 
presentaron dolor del pilar que cedió a los dos meses. Se obtuvo una mejoría clínica significativa en la escala Quick-DASH, con valores preoperatorios de 65.3 y postoperatorios de 21.3 ( $\mathrm{p}=$ 0.0005 [IC95\% 23-67]). Conclusión: Se trata de una técnica quirúrgica con ventajas sobre la técnica abierta tradicional, endoscópica y miniinvasiva sin instrumentación especial, con buenos resultados clínicos.

Palabras clave: Síndrome del túnel del carpo, cirugía mínimamente invasiva.

Nivel de evidencia: IV presented pilar pain that resolved after two months. Clinical improvement with statistical significance was obtained in the Quick-DASH scale, with a change from a preoperative value of 65.3 to a postoperative value of $21.3(\mathrm{p}=0.0005$ [CI95\% 23-67]). Conclusion: This is a surgical technique with advantages over the traditional open technique, the endoscopic technique, and the mini-open technique without special instrumentation.

Keywords: Carpal tunnel syndrome, minimally invasive surgery.

Level of evidence: $I V$

\section{INTRODUCCIÓN}

El síndrome del túnel del carpo (STC) es una neuropatía compresiva que afecta al nervio mediano en la muñeca. Su fisiopatología comprende el aumento de presión intraneural en un espacio cerrado, que a su vez causa isquemia neural y fibrosis del epineuro, hasta muerte axonal. ${ }^{1}$ Los síntomas clásicos son parestesias e hipoestesia constantes o intermitentes en la distribución sensitiva del nervio mediano, respetando el territorio inervado por la rama cutánea palmar. ${ }^{2}$ También es común la interrupción del sueño y, en ocasiones, se puede acompañar de dolor. ${ }^{3}$ Los casos graves se presentan con denervación de la musculatura del pulgar, lo que provoca debilidad y atrofia tenar.

El STC es la forma de compresión nerviosa más común en la población general, con una prevalencia reportada entre 0.1 y $10 \%$ en diferentes series. ${ }^{4}$ Se estima una incidencia anual entre 0.5 y 5.1 por 1,000 habitantes y una acumulativa de $8 \%$. Es más frecuente en mujeres que en hombres, y se presenta de manera más común entre la cuarta y quinta década de la vida, sobre todo en poblaciones trabajadoras. La liberación del túnel del carpo es una de las cirugías de la mano que más se realizan, con cerca de 1.5 por 1,000 habitantes al año. ${ }^{5}$

El diagnóstico del STC se basa en los síntomas y en la exploración física. Éste suele ser muy claro en los pacientes que presentan síntomas nocturnos; además de pruebas de monofilamentos, de discriminación a dos puntos (prueba de Weber) y prueba de Durkhan positivas. ${ }^{2}$ La presencia de las pruebas de Phalen y Tinel positivas, además de la prueba de colapso por rascado aumenta la sensibilidad del diagnóstico hasta $64 \%$, con una especificidad de $99 \% .^{6}$ Los estudios electrodiagnósticos confirman los hallazgos clínicos. ${ }^{7}$

El tratamiento inicial del STC es no-quirúrgico e incluye modificaciones del estilo de vida y uso noc- turno de férulas. La infiltración local con esteroides y el uso oral de AINEs también promueven alivio del cuadro. ${ }^{8}$ Cuando el tratamiento no-quirúrgico falla se considera la cirugía. La descompresión del nervio mediano al dividir el LTC es el tratamiento de elección y se sabe que es la forma más confiable para aliviar los síntomas, por encima de la infiltración de esteroides, terapia física, uso de ortesis y de antiinflamatorios. ${ }^{9}$ Las indicaciones del procedimiento quirúrgico incluyen alteraciones neurológicas, síntomas graves al momento del diagnóstico, atrofia o debilidad muscular tenar y evidencia electrodiagnóstica de debilidad..$^{10}$ Las opciones quirúrgicas incluyen la cirugía convencional con incisión cutánea amplia, las técnicas mínimamente invasivas, las endoscópicas ${ }^{8}$ y las guiadas por ultrasonido. ${ }^{11}$ Sin embargo, la técnica abierta es el método más utilizado, con una alta tasa de éxito y mínimas complicaciones. ${ }^{9}$ Por estudios previos sabemos que la recuperación de la fuerza muscular tras la liberación endoscópica del túnel del carpo se puede esperar a los 16 meses postoperatorios. $^{2}$

El cirujano es quien decide la técnica a utilizar de acuerdo con su entrenamiento y experiencia. Sin importar la técnica empleada, lo más importante es evitar la lesión al nervio mediano o a sus ramas, en especial, la rama cutánea palmar, la rama motora tenar o los nervios digitales comunes. Las dos premisas primordiales para realizar un procedimiento seguro son el conocimiento anatómico preciso del área quirúrgica y el dominio de la técnica elegida. El LTC se puede incidir del lado cubital ${ }^{8}$ o, en ocasiones, del lado radial, ${ }^{12,13}$ cuidando siempre la rama motora tenar. Existe una discusión permanente sobre el método quirúrgico más adecuado, que incluye factores clínicos como el tiempo de recuperación y la prevención de complicaciones, técnicos como la curva de aprendizaje y el uso de instrumental especial; y, por último, logísticos, como costo-efectividad y el tiempo de incapacidad laboral, entre otros..$^{2,14,15}$ 
An Med (Mex) 2020; 65 (1): 9-15

El propósito del presente estudio fue describir una técnica mínimamente invasiva, la cual no requiere instrumentación especial para su realización; además, el objetivo secundario fue revisar los resultados clínicos de pacientes tratados con esta técnica.

\section{MATERIAL Y MÉTODOS}

Es una serie de casos retrospectiva en pacientes manejados por los dos primeros autores, con la técnica descrita por otro de ellos. Se incluyeron todos los pacientes consecutivos: 1) mayores de edad; 2) con diagnóstico de STC a partir de los hallazgos del interrogatorio y la exploración física; 3) que fueron intervenidos de liberación del túnel del carpo primaria con la presente técnica mínimamente invasiva, de julio a diciembre de 2019. Todos los procedimientos se realizaron de acuerdo con los estándares éticos del comité en experimentación humana de la Declaración de Helsinki de 1975, revisada en 2008.

La principal indicación fue pacientes con STC, sin deficiencia muscular tenar. Esta técnica requiere la ausencia de alteraciones anatómicas en el área quirúrgica. Las contraindicaciones para ésta incluyen la liberación previa del túnel del carpo, sin importar la técnica; la presencia de infección o cicatriz previa en el área quirúrgica. El estudio incluyó siete pacientes, uno con intervención bilateral. Seis pacientes del sexo femenino. La edad promedio fue de $44 \pm \mathrm{DE}$ 13.3 años (mín. 24, máx. 65, rango 41). Los siete con dominancia derecha. Seis liberaciones se hicieron en la muñeca derecha y dos en la izquierda.

En cinco se realizó electromiografía, las cuales reportaron dos compresiones severas del nervio mediano, una moderada y en otra no se estadificó el daño neural. En un caso con compromiso bilateral se liberaron ambos lados en un mismo día por solicitud del paciente. En una mujer se realizó la descompresión del nervio mediano en el tercio proximal del antebrazo durante el mismo procedimiento; en otra se efectuó una descompresión del nervio cubital en el codo, con transposición anterior subfascial; finalmente, en otra más se hizo una revisión de primera corredera dorsal por una tenosinovitis estenosante de EPB y APL ipsilateral, ya quirúrgica. El paciente con compromiso bilateral tiene historia de abuso de esteroides; en otros dos había hipotiroidismo controlado; mientras que en otra historia de artritis reumatoide también en control.

El protocolo de seguimiento estándar incluyó al menos tres visitas postoperatorias a las dos, cuatro y ocho semanas. Se utilizaron datos objetivos y subjeti- vos con la finalidad de obtener resultados significativos y clínicamente relevantes. Por un lado, se registraron los resultados de la prueba de Weber pre- y postoperatoria realizada en la punta del dedo pulgar afectado. Además, se llevó a cabo el cuestionario Quick-DASH, evocado según lo descrito por Reynolds y Thirkannad, ${ }^{16}$ que permite la evocación retrospectiva de la condición preoperatoria y después se compararon los resultados con los actuales del Quick-DASH. ${ }^{17}$

El análisis estadístico incluyó una t de Student para comparar la puntuación del Quick-DASH pre- y postoperatoria, además del cambio en la prueba de Weber.

Técnica quirúrgica. Todas las cirugías fueron realizadas por los dos primeros autores. En todos los casos, el paciente se colocó en decúbito supino, con la extremidad a intervenir sobre una extensión lateral de la mesa quirúrgica para apoyar la extremidad superior, con el hombro en abducción de $90^{\circ}$ y el antebrazo en supinación. Se colocó un bloqueo regional infraclavicular en cuatro de los casos y anestesia local en los otros cuatro. Se utilizó isquemia con mango neumático programado a $220 \mathrm{mmHg}$ durante la cirugía. El vaciamiento se realizó con gravedad y compresión leve con las manos del cirujano y del ayudante. En todos los procedimientos se utilizó magnificación de 3.5x. Esta técnica no debe realizarse sin lupas de aumento.

El siguiente paso es, en cierta manera, similar al establecimiento del portal distal en una liberación endoscópica con técnica de Chow. ${ }^{18}$ Primero se identifican la línea de Kaplan y la línea del cuarto

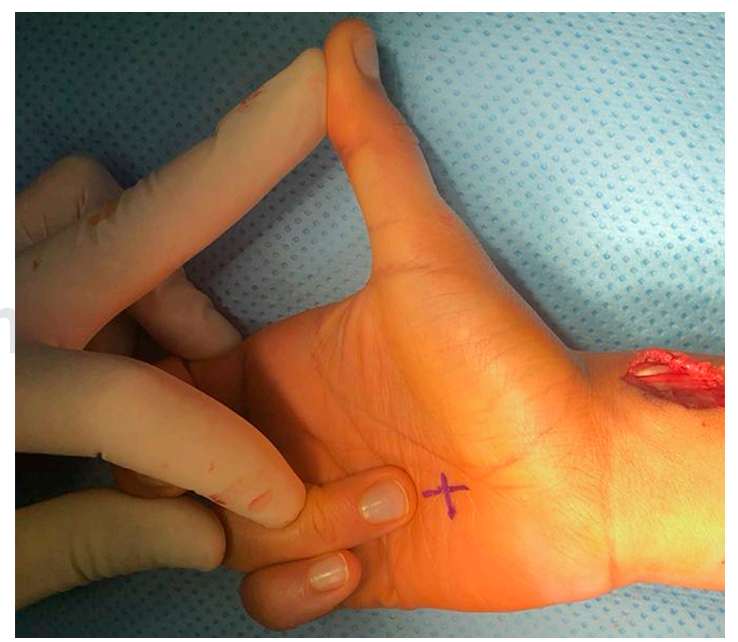

Figura 1: Identificación de la línea de Kaplan y del eje longitudinal del cuarto rayo. 
rayo. Se coloca una marca longitudinal en la piel de aproximadamente $2 \mathrm{~cm}$, la mitad de esta línea va distal a la línea de Kaplan y la mitad proximal a la misma (Figura 1). Se incide la piel con una hoja de

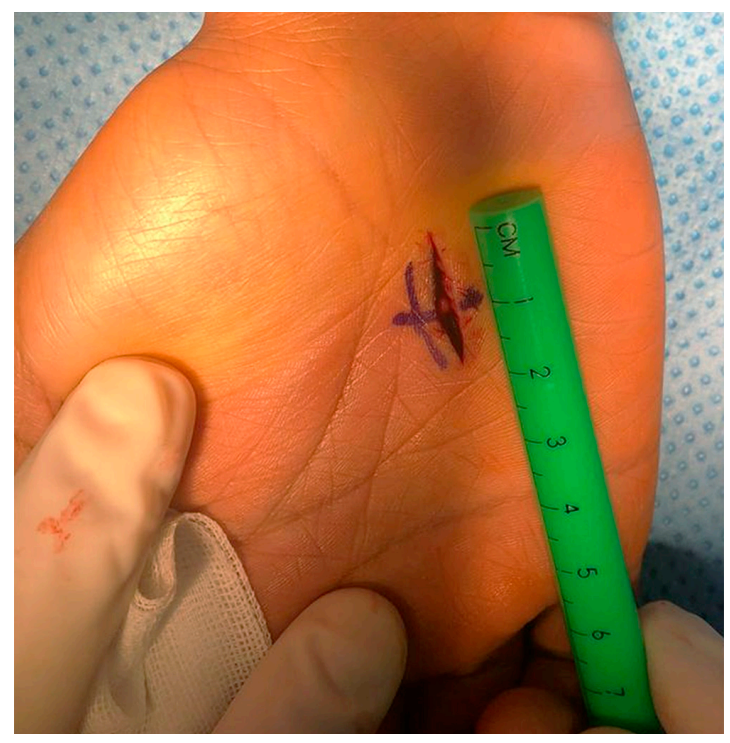

Figura 2: Se demuestra el tamaño de la incisión que se utiliza en esta técnica para realizar la liberación del túnel del carpo. bisturí no. 15, para exponer la fascia palmar, cuyas fibras corren en sentido longitudinal al eje mayor de la extremidad. Se realiza disección roma con tijeras de tenotomía, con lo que se retrae el tejido subcutáneo, después de identificar la fascia se hace una ventana longitudinal igual en dimensiones a la incisión cutánea (Figura 2). Luego, se identifica el plano entre la fascia palmar y el LTC, cuyas fibras son transversales. El LTC se diseca en sentido retrógrado con la tijera de tenotomía, la punta debe dirigirse hacia palmar y el eje de la tijera hacia cubital, recargando la tijera sobre el gancho del ganchoso, de forma similar a una liberación endoscópica en la que el mango de la cuchilla-cámara se recarga en el proceso unciforme. Una vez identificada de forma subcutánea la punta del bisturí proximal al pliegue palmar proximal, se abren las tijeras y se extraen abiertas. Cerrar las tijeras en este paso pone en riesgo las estructuras neurovasculares de la zona. Se introduce nuevamente la tijera cerrada hasta sentir la punta proximal al pliegue de la muñeca y se vuelve a extraer abierta. Esto se repite las veces necesarias para crear un plano adecuado entre la fascia y el LTC en el que pueda entrar la valva de un separador de Senn-Miller. El ayudante retrae con dos valvas de Senn-Miller hacia radial y cubital, mientras que el cirujano sostiene la

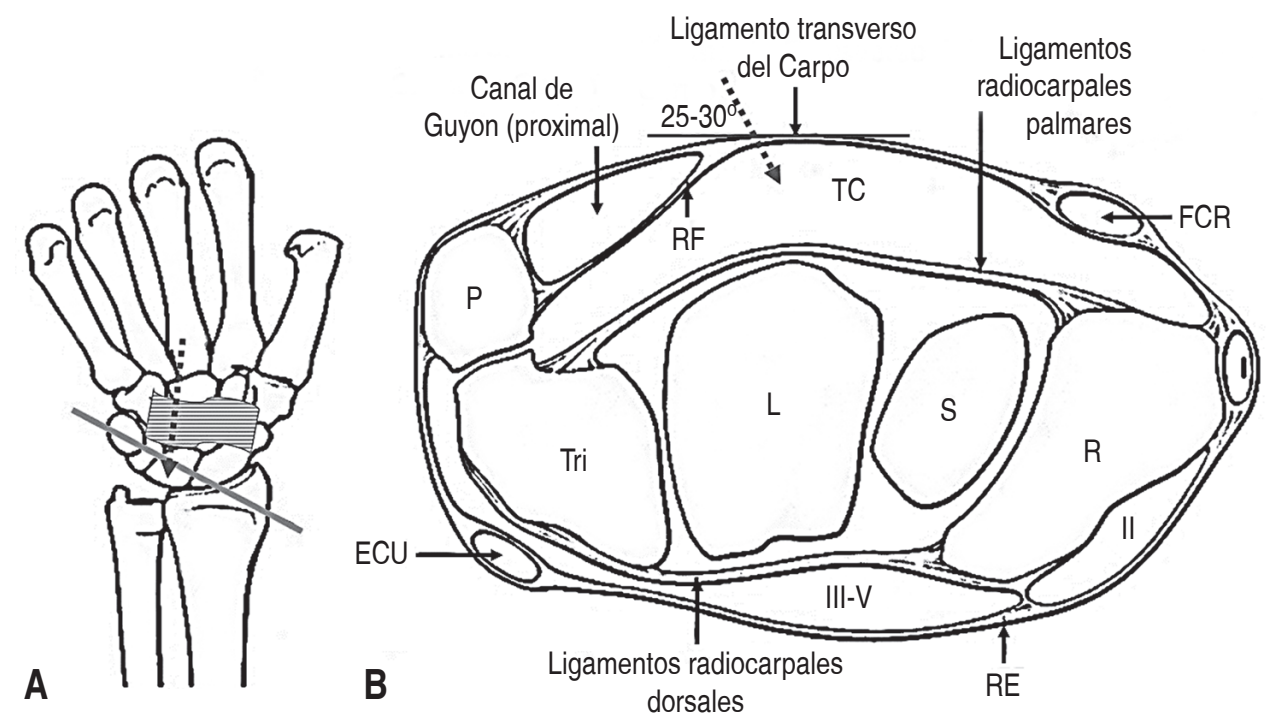

Figura 3: Esquema que indica las relaciones anatómicas del túnel del carpo. A) Dibujo palmar de la muñeca en la que se ilustra con líneas transversales continuas el ligamento transverso y con una flecha punteada se indica el sitio de la incisión. La línea gris muestra el plano de corte para la imagen B. B) corte oblicuo al nivel de la muñeca, en donde se muestra con una flecha punteada el sitio de sección del ligamento transverso del carpo, así como la angulación que debe tener la hoja del bisturí, alejándose del paquete neurovascular cubital, contenido en el canal de Guyon.

$\mathrm{P}=$ pisiformis (pisiforme), $\mathrm{RF}=$ retináculo flexor, $\mathrm{TC}=$ túnel del carpo, $\mathrm{FCR}=$ flexor carpi radialis, Tri $=$ triquetrum (piramidal), $\mathrm{L}=$ lunatum (semilunar), $\mathrm{S}=$ skaphoeidés (escafoides), $\mathrm{R}=$ radio, I = primera corredera extensora, $\mathrm{ECU}=$ extensor carpi ulnaris, III-V = tercer a quinto compartimentos del retináculo extensor, $\mathrm{II}=$ segunda corredera extensora, $\mathrm{RE}=$ retináculo extensor. 
An Med (Mex) 2020; 65 (1): 9-15

valva de otro Senn-Miller en el techo de la incisión y realiza tracción hacia palmar. En este momento el cirujano debe colocarse de frente a la extremidad abducida sobre la mesa de mano, para observar de frente el borde distal del LTC, mientras que se realiza extensión de muñeca entre 15 y 25 grados para una mejor visualización. El cirujano entonces corta de distal a proximal el LTC, sosteniendo el mango del bisturí oblicuo a las fibras de 25 a 30 grados en sentido radial, para evitar lesionar el contenido del canal de Guyon (Figura 3). Los cortes deben ir en sentido anteroposterior, empujando el mango del bisturí hacia el interior del túnel carpiano y hasta sentir la pérdida de resistencia del segmento del LTC incidido. El bisturí no debe deslizarse hacia adelante, porque se pueden lesionar el nervio mediano, el nervio digital para el anular o los tendones flexores. Tras seccionar la totalidad del LTC, se verifica el grosor de la fascia antebraquial, si existiera una banda tensa de la fascia se corta con bisturí o con tijeras de tenotomía. Se retira la isquemia y se realiza hemostasia con coagulación mono o bipolar. Se cierra la herida con puntos de colchonero horizontal con sutura de monofilamento no absorbible y se cubre con gasas y venda simple estériles.

\section{RESULTADOS}

Los siete pacientes de este estudio recibieron un seguimiento mínimo postoperatorio a dos meses. En todos los casos los síntomas cedieron por completo, excepto en la paciente número 4 , en quien hubo una mejoría importante tanto objetiva (Weber de 8 a $5 \mathrm{~mm}$ ), fuerza muscular 5/5 en la escala Medical Research Council, ${ }^{19}$ como subjetiva (Quick-DASH); sin embargo, persiste con parestesias en el territorio inervado por el mediano. En el paciente número 1, operado de forma bilateral, se presentó dolor del pilar en ambas muñecas, que se resolvió a los dos meses. En la número 7, a quien se le hizo la liberación del nervio mediano en el tercio proximal del antebrazo, se presentó un síndrome del nervio interóseo anterior, que cedió a los dos meses postoperatorios. No hubo necesidad de cirugía de revisión en ningún caso.

Cuando se llevó a cabo el análisis estadístico del cambio de puntuación del Quick-DASH preoperatorio versus postoperatorio se encontró una mejoría importante en todos, excepto en la número 7 , que cursó con tenosinovitis estenosante del pulgar ipsilateral al momento de realizar el Quick-DASH postoperatorio. $\mathrm{Al}$ analizar el promedio de resultados del
Tabla 1: Resultados funcionales finales.

\begin{tabular}{|c|c|c|}
\hline $\begin{array}{l}\text { Evaluación subjetiva/ } \\
\text { objetiva }\end{array}$ & $\begin{array}{l}\text { Promedio (mediana) } \\
\text { 土DE (mín.-máx.) }\end{array}$ & $\begin{array}{l}\text { t de Student } \\
\text { p (IC 95\%) }\end{array}$ \\
\hline Quick-DASH pre & $65.3(63.63) \pm 16.3(34-86)$ & $0.0005(23.6-67.3)$ \\
\hline Quick-DASH post & $21.3(10.2) \pm 23.7(4.5-75)$ & \\
\hline Weber pre (pulgar) & $3.9(3) \pm 1.7(3-8)$ & $0.377(-0.8-2.1)$ \\
\hline Weber post (pulgar) & $3.2(3) \pm 0.6(3-5)$ & \\
\hline
\end{tabular}

cuestionario Quick-DASH pre- y postoperatorio se encontró una mejoría media de 44 puntos, se tuvo una diferencia con significancia estadística entre las mediciones con un valor de $\mathrm{p}=0.0005$ (IC95\% 2367). En cuanto a los cambios en la prueba de Weber realizada en la punta del pulgar de la mano afectada, el análisis estadístico no arrojó ningún resultado significativo, $\mathrm{p}=0.377$ (IC95\% -0.8-2.1); no obstante, clínicamente en todos los casos, hubo mejoría en la prueba de discriminación a dos puntos (Tabla 1).

\section{DISCUSIÓN}

La liberación del túnel del carpo es una de las cirugías más comunes en cirugía de la mano. ${ }^{20}$ Existen diferentes técnicas quirúrgicas disponibles para tratar la neuropatía compresiva del nervio mediano en la muñeca, pero la decisión final de la técnica a emplear recae primordialmente en el cirujano.

Entre las técnicas disponibles la más accesible es la clásica, en la que se incide 2-4 mm cubital al pliegue tenar, desde la línea de Kaplan, hasta llegar al pliegue de la muñeca, y que se puede extender de forma proximal a éste entre el FCU y el PL. ${ }^{21}$ Esta técnica y sus múltiples modificaciones tienen la ventaja de mostrar por completo el LTC, así como las estructuras dentro del túnel carpiano; además, se puede identificar y proteger la rama motora tenar. ${ }^{8}$ También se puede utilizar en caso de realizar sinovectomía o en cirugías de revisión en las que es necesario encontrar tejido sano o realizar neurólisis. ${ }^{9}$

El uso de técnicas endoscópicas ha mejorado el tratamiento en términos de mínima invasión, y tiene la ventaja de que evita la formación de una cuerda de arco de los tendones flexores y la subluxación hacia palmar hasta la cicatriz cutánea. Las técnicas más conocidas son las descritas por Agee, Chow y Menon. ${ }^{2,8}$ Todas ellas requieren una curva de aprendizaje importante; además, el cirujano debe estar familiarizado con las técnicas endoscópicas y artroscópicas, 
An Med (Mex) 2020; 65 (1): 9-15

y saber reconocer las estructuras anatómicas en la pantalla. A diferencia de lo que podría pensarse, el dolor postoperatorio del pilar no disminuye con esta técnica y se presentó con la misma frecuencia que en otras series reportadas. ${ }^{22}$

Se han descrito técnicas mínimamente invasivas de dos incisiones que pueden sustituir a las técnicas endoscópicas, lo que permite una adecuada visualización para lograr la sección completa del LTC. La técnica de Wilson describe el uso de dos incisiones, una transversal justo proximal al pliegue de muñeca, y una longitudinal muy parecida a la que se describe en esta técnica. Creemos que la incisión proximal no es necesaria, pues con una sola incisión distal se consigue la sección completa del LTC, el objetivo último del procedimiento. ${ }^{23}$ Otra técnica recientemente descrita usa dos incisiones, muy parecidas a la técnica endoscópica de dos portales; pero ésta requiere instrumental especial para su realización. ${ }^{24}$

Con el advenimiento de las técnicas endoscópicas, se describieron otras mínimamente invasivas para evitar el uso de equipo de endoscopia. En la técnica de Bromley, parecida a la que se presenta en este trabajo, la visualización es teóricamente comparable con una endoscopia, ${ }^{25}$ pero el corte del LTC se realiza de forma retrógrada sólo con tijeras de tenotomía. Consideramos que se trata de un gesto quirúrgico riesgoso, ya que es difícil controlar que el contenido del túnel del carpo se interponga entre las hojas de la tijera, por lo que existe el riesgo de lesión nerviosa o tendinosa. En otras técnicas mínimamente invasivas, el procedimiento se realiza a ciegas y, además, se requiere de instrumentación especial. ${ }^{26-28}$ En particular, en la técnica descrita en este trabajo, se usa instrumental común de cirugía de mano, tres separadores de Senn-Miller, unas pinzas de disección Adson con dientes, tijeras de tenotomía Stevens curvas y un mango de bisturí no. 3 con una hoja no. 15. Todo lo anterior es suficiente para realizar el procedimiento.

\section{CONCLUSIÓN}

La técnica mínimamente invasiva presentada en este trabajo otorga una ventaja sobre la técnica abierta tradicional, ya que evita la formación de una cuerda de arco de los tendones flexores y el nervio mediano hacia palmar; además, presenta una cicatrización cutánea más rápida. También proporciona ventajas sobre la técnica endoscópica, ya que requiere menor tiempo de preparación del campo quirúrgico, menor tiempo del procedimiento per se y menos instrumentación especial, lo que disminuye los costos. El ciruja- no que empiece a realizar esta técnica debe tener un conocimiento anatómico y quirúrgico pleno, además de respetar en todo momento los pasos descritos. Los resultados clínicos son similares a las otras técnicas en cuanto a la aparición de complicaciones, tales como dolor del pilar y a la resolución de los síntomas clínicos e interrupción del deterioro neural.

\section{BIBLIOGRAFÍA}

1. Mackinnon SE, Novak CB. Compression Neuropathies. In: Wolfe SW, Hotchkiss RN, Pederson WC, Kozin SH, editors. Green's operative hand surgery. 6th ed. Philadelphia, PA: Elsevier Inc; 2011.

2. Farías E, Valencia DF, León SR, Avilés LA, Espinosa A. Functional results after carpal tunnel endoscopic release on a public health out-patient surgical campaign. Investig en Discapac. 2018; 7 (3): 79-90.

3. Bickel KD. Carpal tunnel syndrome. J Hand Surg. 2010; 35 (1): 147-152.

4. Parisi DM, Trumble TE. Wrist and hand reconstruction. In: Vaccaro AR, editor. Orthopaedic knowledge update home study syllabus. 8th ed. Rosemont IL: American Academy of Orthopaedic Surgeons; 2005. pp. 351-373.

5. Davidge KM, Sammer DM. Median nerve entrapment and injury. In: Mackinnon SE, Yee A, editors. Nerve Surgery. New York, N.Y.: Thieme; 2015. pp. 207-250.

6. Cheng CJ, Mackinnon-Patterson B, Beck JL, Mackinnon SE. Scratch collapse test for evaluation of carpal and cubital tunnel syndrome. J Hand Surg Am. 2008; 33: 1518-1524.

7. Rempel D, Evanoff B, Amadio PC, de Krom M, Franklin G, Franzblau A et al. Consensus criteria for the classification of carpal tunnel syndrome in epidemiologic studies. Am J Public Health. 1998; 88: 1447-1451.

8. Mendoza MM, Hernández BR, Farías CE. Tratamiento qirúrgico del síndrome del túnel del Carpo. In: Ibarra LG, Ibarra Ponce de León JC, editors. Cirugía para el tratamiento de la discapacidad. México, D.F.: Alfil; 2014. pp. 223-246.

9. Keith MW, Masear V, Amadio PC, Andary M, Barth RW, Graham B et al. Treatment of carpal tunnel syndrome. J Am Acad Orthop Surg. 2008; 17: 397-405.

10. Maggard M, Harness N, Chang W, Parikh J, Asch S, Nuckols $\mathrm{T}$ et al. Indications for performing carpal tunnel surgery: clinical quality measures. Plast Reconstr Surg. 2010; 126 (1): 169-179.

11. Buncke G, McCormack B, Bodor M. Ultrasound-guided carpal tunnel release using the manos CTR system. Microsurgery. 2013; 33 (5): 362-366.

12. Tsai TM, Laurentin-Perez LA, Wong MS, Tamai M. Ideas and innovations: radial approach to carpal tunnel release in conjunction with thumb carpometacarpal arthroplasty. Hand Surg. 2005; 10 (1): 61-66.

13. Chan CM, Farías Cisneros E, Tsai TM. Trapeziometacarpal joint arthroplasty of the thumb without osseous tunnels and concurrent carpal tunnel release via a radial approach; surgical technique and results. Surg J. 2019; 5: e120-125.

14. Herrera J, Ceja B, Hernández J, Sesma R, Gargollo C. Carpal tunnel release with minimal incisions. Evaluation of the technique in fresh cadaver models. Acta Ortop Mex. 2013; 27 (4): 260-264.

15. Kim P, Lee H, Kim T, Jeon I. Current approaches for carpal tunnel syndrome. Clin Orthop Surg. 2014; 6 (3): 253-257.

16. Reynolds N, Thirkannad SM. The recall DASH score-a novel research tool. Hand Surg. 2013; 18 (1): 11-14. 
An Med (Mex) 2020; 65 (1): 9-15

17. Sambandam S, Priyanka P, Gul A, Ilango B. Critical analysis of outcome measures used in the assessment of carpal tunnel syndrome. Int Orthop. 2008; 32 (4): 497-504.

18. Chow J. Endoscopic release of the carpal ligament: a new technique for carpal tunnel syndrome. Arthroscopy. 1989; 5: 19-24.

19. Paternostro-Sluga T, Grim-Stieger M, Posch M, Schuhfried O, Vacariu G, Mittermaier C et al. Reliability and validity of the Medical Research Council (MRC) scale and a modified scale for testing muscle strength in patients with radial palsy. J Rehabil Med. 2008; 40 (8): 665-671.

20. Agee JM, Peimer CA, Pyrek JD, Walsh WE, Bickel KD, Jebson PJL et al. Carpal tunnel syndrome. J Hand Surg. 2010; 35 (2): 165-171.

21. Taleisnik J. The palmar cutaneous branch of the median nerve and the approach to the carpal tunnel. An anatomical study. J Bone Joint Surg Am. 1973; 55 (6): 1212-1217.

22. Vasiliadis HS, Nikolakopoulou A, Shrier I, Lunn MP, Brassington R, Scholten RJP et al. Endoscopic and open release similarly safe for the treatment of carpal tunnel syndrome. A systematic review and meta-analysis. PLoS One. 2015; 10 (12): 1-16.

23. Wilson K. Double incision open technique for carpal tunnel release: an alternative to endoscopic release. J Hand Surg Am. 1994; 19 (6): 907-912.

24. Gaba S, Bhogesha S, Singh O. Limited incision carpal tunnel release. Indian J Orthop. 2017; 51 (2): 192-198.

25. Bromley G. Minimal-incision open carpal tunnel decompression. J Hand Surg Am. 1994; 19 (1): 119-120.

26. Pagnanelli DM, Barrer SJ. Carpal tunnel syndrome: surgical treatment using the paine retinaculatome. J Neurosurg. 1991; 75 (1): 77-81.

27. Hwang P, Ho C. Minimally invasive carpal tunnel decompression using the KnifeLight. Neurosurgery. 2007; 60 (2 Suppl 1): ONS162-1689.

28. Lee WP, Schipper BM, Goitz RJ. 13-year experience of carpal tunnel release using the indiana tome technique. J Hand Surg Am. 2008; 33 (7): 1052-1056. 